\title{
The $r-l$ square point process: The effect of coordinated multipoint joint transmission
}

\author{
Iyad Lahsen Cherif ${ }^{1}$, Lynda Zitoune ${ }^{2,1}$, Véronique Vèque ${ }^{1}$ \\ ${ }^{1}$ Signals and Systems Laboratory, (L2S, UMR CNRS 8506) \\ CNRS - CentraleSupélec - Université Paris-Sud \\ 3, Rue Joliot-Curie, 91192 Gif sur Yvette, France. \\ \{iyad.lahsen-cherif, Veronique.VEQUE\} @l2s.centralesupelec.fr \\ ${ }^{2}$ Department of Systems Engineering, ESIEE-Paris \\ 2, boulevard Blaise Pascal, 93162 Noisy le Grand, France. \\ lynda.zitounedesiee.fr
}

\begin{abstract}
A 1-tier network composed by multimode low power nodes (LTE/Wifi) is considered as a cost-efficient solution for operators to improve services in poorly or uncovered rural areas. Using an interference coordination technique, network performance can be further improved. Stochastic geometry gives a set of tools to model the location of base stations and user equipments in such wireless networks. Using a spatial model we analyse the network performance in terms of coverage probability and data rate. To realistically model multimode node locations, a new point process model, called the $r-l$ square point process (p.p.), is used in this work. The model of downlink communication including the coordination technique is developed and it allows to evaluate the system performances in term of coverage probability and throughput. Results show that cooperation among nodes improves the network performance.
\end{abstract}

Index Terms-LTE/Wifi; CoMP; Stochastic geometry;

\section{INTRODUCTION}

According to ITU statistics, there are over 6.8 billion active subscribers of cellular networks [1]. However, nowadays many rural areas all over the world are still lacking of the connectivity service. This is due to two main factors : the installation cost of macro base stations (BS) and the non existence of any kind of wired infrastructure in such areas. An innovative and a cost-efficient solution for operators to provide services to poorly-covered or uncovered areas is the deployment of outdoor multimode femtocells. A multimode femtocell is a low power node (LPN) operating on both cellular (4G/LTE) and Wi-Fi technologies. This new generation of femtocells are expected to offload the cellular network traffic on Wi-Fi bands to improve the capacity in case of dense networks [2].

As the position of nodes plays an important role in wireless communications, the multimode femtocell location should be defined carefully. LPNs must not be lied too close together to reduce the interference mainly at the cell edge. But at the same time, they should not be further apart than Wi-Fi transmission range. The aim is to form a Wi-Fi mesh network using the Wi$\mathrm{Fi}$ interfaces of these nodes, and to use it as an access network to the macro base station. As a consequence, the underlying Wi-Fi mesh network extends the coverage of the macro BS to consider far mobiles users, as shown in figure 1.

978-1-4799-5344-8/15/\$31.00 (c) 2015 IEEE
To deploy such a network in rural zone, it is expected that multimode base stations are outsourced or managed by some Virtual Operators (VOP) such as city hall, schools or compagnies. So that, the scenario of figure 1 considered here is completely different from classical deployment of femtocells which intends to increase the capacity of macro cellular network in urban regions. In such cases, the position of the femtocell BS is user-dependent leading to dense network. In stochastic geometry, Poisson point process is intensively used to study the performance of such deployment in terms of coverage/outage probability, and bit rate [3]-[11]. The Poisson Point Process model is used as it is more trackable than others point processes of stochastic geometry.

To the opposite to PPP model, and to consider the scenario of the figure 1, [12] proposed a new point process called $r$ - $l$ square process to model the position of the multimode femtocell BSs and to evaluate the coverage probability depending on the distance that separates the nodes. $r-l$ square process allows a correlation between nodes defined by Wi-Fi communication ranges and deployment policies specified by the Virtual Operators responsible of the deployment of these base stations in the rural region.

However, like femtocell networks in rural region used to increase the capacity, our scenario suffers from interference. This is mainly due to the difference between the coverage of both cellular (4G/LTE) and WiFi technologies. For example, commercial outdoor femtocell base stations ensures a coverage about $750 \mathrm{~m}$ with a transmission power of 5 watts, whereas outdoor WiFi access points cover only $250 \mathrm{~m}$. Consequently, when many multimode LPNs are used to cover a region, a severe interference can be generated.

Hence, interference is one of the challenges facing the femtocells deployment which affect considerably the network performance. Recently, the Coordinated Multi-Point techniques (CoMP) [7], [13]-[15] has been attracting more attention. This coordination technique intends to reduce interference and improve throughput of cell edge users. Joint Transmission (JT) and Coordinated Beamforming/Scheduling $(\mathrm{CB} / \mathrm{CS})$ are two ways to perform CoMP.

The aim of this work is to improve the coverage probability 


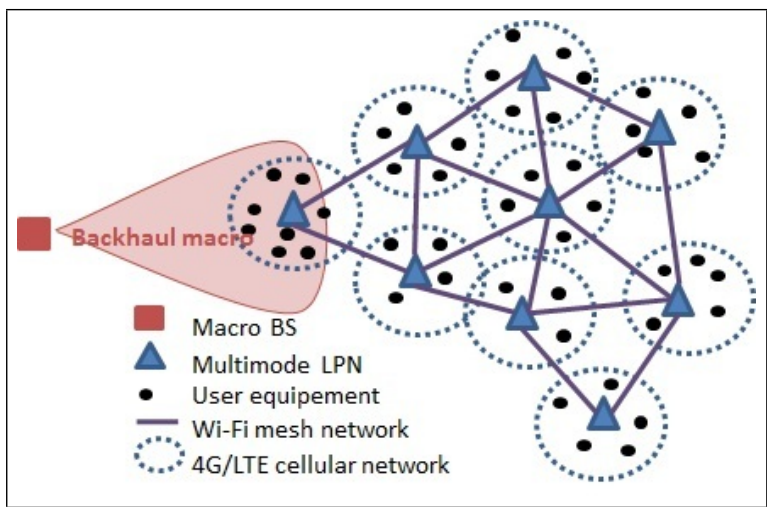

Fig. 1: Femtocell and Wi-Fi mesh network architecture

of a 1-tier network composed of low power nodes (scenario in fig 1) using a coordination technique. To realistically model femtocell locations the $r-l$ square process is used [12]. The model of downlink communication including the coordination technique is developed and allows us to evaluate the system performance in terms of coverage probability and throughput.

This paper is structured as follows. We first introduce in section II some related work on cellular network modeling using stochastic geometry and on CoMP techniques. In section III, we recall the definition of $r-l$ square p.p. model and detail our new analytical model of coordination based on $r-l$ square p.p.. We will evaluate the resulting model by simulation and discuss the results in section IV. We will conclude the paper in section $\mathrm{V}$ and give some remarks to improve the model in the future works.

\section{RELATED WORK}

In this section, we first introduce some related work on stochastic geometry models. Next, we present new interference mitigation approaches using coordination.

\section{A. Point Processes}

Many types of point processes (p.p.) [3]-[11] are used in literature to model wireless network. They can be classified as either irregular or regular processes.

a) Irregular point processes: Poisson p.p. is the most used example of irregular p.p. [3]-[8].

Poisson Point Process is the point process with zero attraction, where points are distributed independently with no relationship among each other. It is characterized by two properties: the number of points in disjoint bounded sets are independent and have a Poisson distribution.

In [3], the coverage probability and the achievable rate in a $K$-tier network are evaluated for femtocells operating in closed and open access modes. The max-SINR connectivity model is used. In [4], the interference in homogeneous and heterogeneous network is characterized by approximating the interference using a Gamma distribution. In those works, analysis is performed using the Laplace Transform (LT) and was limited to a Rayleigh fading channel. However, alternative tools can be used to simplify calculations and obtain more general results, considering a more general fading distribution. In this context, we can cite Factorial Moment Measure [6], Moment Generating Function [5], Plancherel-Parseval theorem [8].

Whereas Poisson p.p. is recognized as a tractable modeling tool, it does not match to the realistic cellular deployment and a lot of works were interested in regular p.p.. In this p.p. type, there is neither attraction nor repulsion between points of the process.

b) Regular point processes : Martérn Point Processess are Hard Core p.p. firstly introduced by Matérn in [16]. Matérn p.p. are models where points repel each other. They are constructed from a Poisson p.p.. Two types of Martérn Hard Core p.p. are considered, Matérn I and Matérn II depending on the selection criterion of the points. In Matérn I all points with a neighbor within the threshold distance $\delta$ are deleted from the process. In Matérn II each point has a uniform mark between 0 and 1, and a node is deleted only if there is another point within distance $\delta$ with a smaller mark. Matérn p.p. is not analytically tractable due to the non-independent nature of points. Lower bounds of the coverage probability in a conventional network (1-tier) are provided in [17] when using Matérn type II. Another regular process type is called Ginibre point process [9]-[11]. It is a determinantal point process which induces a repulsion between points. This repulsion is interpreted by the probability density to place points. In fact, the probability to draw a point at the same position of an already drawn point is zero. This probability increases by increasing the distance from every existing points. The authors of [9] derive the coverage probability in a 1-tier network and promote its asymptotic property. They extend their work to an heterogeneous network in [10]. [11] used the Palm measure and reduced second moment approaches to derive the mean and variance of the interference.

All these p.p. are either not fit to model the real deployment of nodes or difficult to analyze.

\section{B. Coordinated Multi-Point: CoMP}

CoMP is a coordination/cooperation technique used to reduce interference and hence increases the network throughput. This coordination/cooperation is performed between BSs by exchanging data: Joint Transmission (JT) or information: Coordination Beamforming/Scheduling (CB/CS).

In JT, users receive multiple copies of the same data from different BSs in the coordinated set, and the signal received from BSs outside the coordinated set is seen as interference. [13] characterizes the SINR distribution and discusses some practical design problems. It concludes that increasing the BS density while fixing the cooperation radius improves the SINR. Therefore the gain of cooperation, in terms of coverage, increases with the path loss exponent. [14] evaluates the coverage probability of a Poisson p.p. heterogeneous network under different connectivity models. It concludes that the $n$ strongest BS connectivity model (the $n$ BSs with strongest received signal) is better than the $n$-nearest $\mathrm{BS}$ one (the nearest $\mathrm{BS}$ from each tier). In $\mathrm{CB} / \mathrm{CS}$, only beamforming vectors 
are shared between coordinated BSs. The effect of non ideal backhaul network is studied in [15]. In fact, the performance metrics decrease linearly with the overhead delay. [7] showed that limited feedback reduces the gain of coordination and it proposes an adaptative bit allocation to overcome this problem. It is proved in [18], that the CoMP-JT strategy offers better performance than $\mathrm{CB} / \mathrm{CS}$. All cited works have considered a Poisson p.p..

The improvement in the network performance are approved in [19] using a realistic urban scenario. Depending on the difference between the received signal strength of the serving cell and the coordinated cells an interference map is constructed. The CoMP gain is then derived using the interference map.

In this work, we use the $r$-l square p.p. which is more appropriate to our scenario. We evaluate the coverage probability and the rate of CoMP JT when nodes are distributed according to the $r$ - $l$ square p.p.. We assume an ideal backhaul to share data.

In the next section, we describe the used spatial point process and explain the motivation behind it.

\section{SySTEM MODEL}

We consider a 1-tier network composed of low power nodes such as picocells and femtocells. Nodes are multimode and operate in open access modes. Mesh network is used to connect LTE/Wifi nodes.

\section{A. r-l square Point Process}

Poisson p.p. generates nodes independently in the plane, which leads to many problems such as uncovered regions or nodes very close to each other strongly interfering between each other. To overcome those problems, a new point process, called $r-l$ square point process has been proposed in [12]. It is built as follows: the plane $\left(\mathbb{R}^{2}\right)$ is divided into squares of the sizes $r \times r$. In each $r \times r$ square, a new sub-square of size $l \times l$ (with $0 \leq l \leq r$ ) is placed. A point is uniformly distributed in each sub-square. Those points represent the nodes. When $l<$ $r$, this process is a Hard Core point process, as the points can not lie at a distance less than $r$-l. Hence, this model imposes that two points in adjacent squares can not be too close to each other (which reflects the real deployment of nodes and overcomes problems of the Poisson p.p.). Mobile users are set according to a Poisson p.p. in the plane. Illustration of this point process is given in figure 2 .

In the following, we focus on the downlink and we evaluate the performance in terms of coverage probability, under the $r-l$ square model described above when coordination in particular CoMP-JT is applied between nodes to mitigate the interference. We assume an ideal Wi-Fi mesh network to transmit all duplicated signals without overloading links.

\section{B. Performance evaluation}

CoMP-JT is a cooperation technique used to reduce interference. User equipment receives data not only from its serving BS but also from all BSs in the coordinated set. Signals

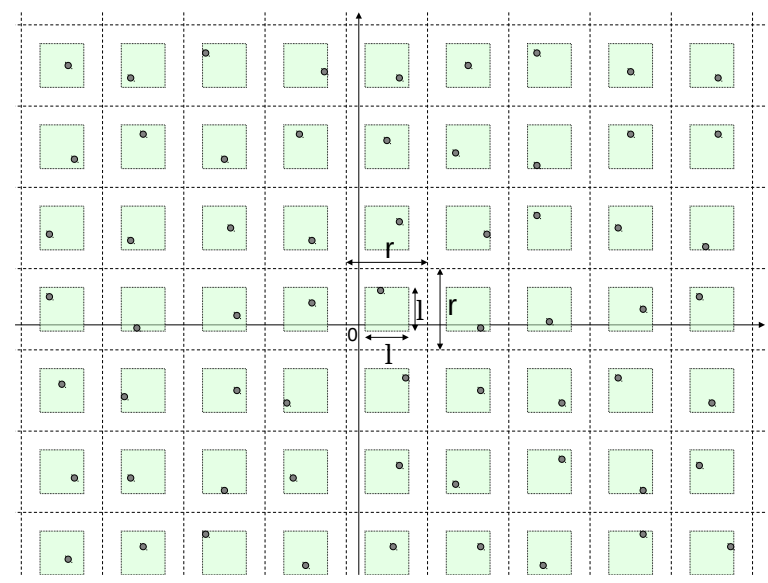

Fig. 2: Construction of the $r-l$ square point process

received from nodes outside the coordinated set are seen as interference. This is illustrated by the following equation 1 .

The received signal at the typical user equipment $u$ is:

$$
y=\underbrace{\sum_{X_{i} \in \mathcal{C}} \sqrt{p_{t}} \xi_{i} \sqrt{l\left(\left\|X_{i}-u\right\|\right)}}_{\text {Useful signal }}+\underbrace{\sum_{X_{j} \in \Phi \backslash \mathcal{C}} \sqrt{p_{t}} \xi_{j} \sqrt{l\left(\left\|X_{j}-u\right\|\right)}}_{\text {Interference }}+\underbrace{N}_{\text {Noise }}
$$

where $\Phi$ is the $r$ - $l$ square p.p., $p_{t}$ is the transmission power of nodes (it is assumed to be the same for all nodes), $\left(\xi_{i}\right)_{i}$ are i.i.d $\sim \mathcal{C N}(0,1)$ Gaussian random variables with mean 1 modeling fading, $l($.$) is the path loss function and N \sim \mathcal{C N}\left(0, \sigma^{2}\right)$ is an additive white Gaussian noise (AWGN). $\mathcal{C}$ is the coordinated set and is defined as follows:

$$
\mathcal{C}=\left\{X_{i} \in \Phi \text { s.t. } X_{i} \in B_{u}(d)\right\}
$$

Nodes not in the coordinated set $(\Phi \backslash \mathcal{C})$ act as interferers. In other words, the coordinating BSs are the BSs inside the ball $B_{u}(d)$ centered at $u$ and radius $d$. All BSs outside the ball act as interferers. Figure 3 illustrates examples of two coordinated sets with different sizes. Solid lines indicate the useful or duplicated signals received by the mobile user $u$ located at the center of the circle.

Thus, the SINR is given by

$$
\operatorname{SINR}=\frac{\left|\sum_{X_{i} \in \mathcal{C}} \sqrt{p_{t}} \xi_{i} \sqrt{l\left(|| X_{i}-u \|\right)}\right|^{2}}{\sum_{X_{j} \in \Phi \backslash \mathcal{C}} p_{t}\left|\xi_{j}\right|^{2} l\left(\left\|X_{j}-u\right\|\right)+\sigma^{2}}
$$

Proposition 1: The coverage probability under JT-CoMP is given by:

$$
\begin{aligned}
p_{c}(T) & =\operatorname{Pr}(\operatorname{SINR}>T) \\
& =\prod_{j} \mathbb{E}\left[\left(\frac{1}{1+T \frac{l\left(\left\|X_{j}-u\right\||| \mathbb{1}_{\left\{X_{j} \notin B_{u}(d)\right\}}\right.}{\sum_{i} l\left(\| X_{i}-u|| \mathbb{1}_{\left\{X_{i} \in B_{u}(d)\right\}}\right.}}\right)\right] \\
& \times \mathbb{E}\left[\exp \left(-T \frac{\sigma^{2}}{\sum_{X_{i} \in \mathcal{C}} p_{t} l\left(\left\|X_{i}-u\right\|\right)}\right)\right]
\end{aligned}
$$




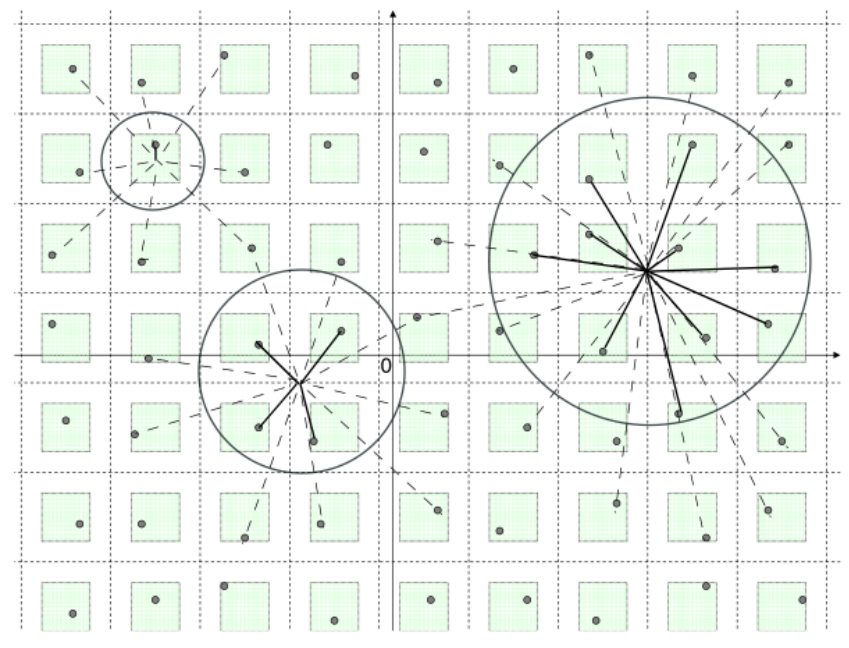

Fig. 3: Illustration of the coordinated set $B_{u}(d)$ : The solid lines represent the useful signal received by a mobile user from the BSs inside $B_{u}(d)$ and the dashed lines represent the interference

Proof 1:

$$
\begin{aligned}
p_{c}(T) & =\operatorname{Pr}(\operatorname{SINR}>T) \\
& =\operatorname{Pr}\left\{\frac{\left|\sum_{X_{i} \in \mathcal{C}} \sqrt{p_{t}} \xi_{i} \sqrt{l\left(\left\|X_{i}-u\right\|\right.}\right|^{2}}{\sum_{X_{j} \in \Phi \backslash \mathcal{C}} p_{t}\left|\xi_{j}\right|^{2} l\left(\left\|X_{j}-u\right\|\right)+\sigma^{2}}>T\right\} \\
& =\operatorname{Pr}\left\{\left|\sum_{X_{i} \in \mathcal{C}} \sqrt{p_{t}} \xi_{i} \sqrt{l\left(\left\|X_{i}-u\right\|\right)}\right|^{2}\right. \\
>T & \left.\left(\sum_{X_{j} \in \Phi \backslash \mathcal{C}} p_{t}\left|\xi_{j}\right|^{2} l\left(\left\|X_{j}-u\right\|\right)+\sigma^{2}\right)\right\} \\
& \stackrel{(a)}{=} \mathbb{E}\left[\exp \left(-T \frac{\sum_{X_{j} \in \Phi \backslash \mathcal{C}} p_{t}\left|\xi_{j}\right|^{2} l\left(\left\|X_{j}-u\right\|\right)+\sigma^{2}}{\sum_{X_{i} \in \mathcal{C}} p_{t} l\left(\left\|X_{i}-u\right\|\right)}\right)\right] \\
& =\mathbb{E}\left[\exp \left(-T \frac{\sum_{X_{j} \in \Phi \backslash \mathcal{C}} p_{t}\left|\xi_{j}\right|^{2} l\left(\left\|X_{j}-u\right\|\right)}{\sum_{X_{i} \in \mathcal{C}} p_{t} l\left(\left\|X_{i}-u\right\|\right)}\right)\right] \\
& \times \mathbb{E}\left[\exp \left(-T \frac{\sigma^{2}}{\sum_{X_{i} \in \mathcal{C}} p_{t} l\left(\left\|X_{i}-u\right\|\right)}\right)\right]
\end{aligned}
$$

where (a) follows the hyper exponential property. The first term is as follows:

$$
\begin{aligned}
& \mathbb{E}\left[\exp \left(-T \frac{\sum_{X_{j} \in \Phi \backslash \mathcal{C}} P_{t}\left|\xi_{j}\right|^{2} l\left(\left\|X_{j}-u\right\|\right)}{\sum_{X_{i} \in \mathcal{C}} P_{t} l\left(\left\|X_{i}-u\right\|\right)}\right)\right] \\
& =\mathbb{E}\left[\exp \left(-T \frac{\sum_{j}\left|\xi_{j}\right|^{2} l\left(\left\|X_{j}-u\right\|\right) \mathbb{1}_{\left\{X_{j} \notin B_{u}(d)\right\}}}{\sum_{X_{i} \in \mathcal{C}} l\left(\left\|X_{i}-u\right\|\right)}\right)\right] \\
& =\mathbb{E}\left[\prod_{j} \exp \left(-T \frac{\left|\xi_{j}\right|^{2} l\left(\left\|X_{j}-u\right\|\right) \mathbb{1}_{\left\{X_{j} \notin B_{u}(d)\right\}}}{\sum_{X_{i} \in \mathcal{C}} l\left(\| X_{i}-u||\right)}\right)\right] \\
& \stackrel{(b)}{=} \mathbb{E}\left[\prod_{j}\left(\frac{1}{1+T \frac{l\left(\left\|X_{j}-u\right\|\right) \mathbb{1}_{\left\{X_{j} \notin B u(d)\right\}}}{\sum_{X_{i} \in \mathcal{C}} l\left(\| X_{i}-u||\right)}}\right)\right] \\
& =\mathbb{E}\left[\prod_{j}\left(\frac{1}{1+T \frac{l\left(\left\|X_{j}-u\right\|\right) \mathbb{1}_{\left\{X_{j} \notin B_{u}(d)\right\}}}{\sum_{i} l\left(\left\|X_{i}-u\right\|||\right) \mathbb{1}_{\left\{X_{i} \in B_{u}(d)\right\}}}}\right)\right] \\
& =\prod_{j} \mathbb{E}\left[\left(\frac{1}{1+T \frac{l\left(\left\|X_{j}-u\right\|\right) \mathbb{1}_{\left\{X_{j} \notin B_{u}(d)\right\}}}{\sum_{i} l\left(|| X_{i}-u \|\right) \mathbb{1}_{\left\{X_{i} \in B_{u}(d)\right\}}}}\right)\right]
\end{aligned}
$$

where (b) from the fact that $\left(\left|\xi_{i}\right|^{2}\right)_{i}$ are exponentially distributed.

Remark 1: In the Interference limited (free noise) regime, the coverage probability is independent from the transmit power of the LPNs.

The rate can be derived from the coverage probability by the following formula:

$$
\begin{aligned}
R & =\mathbb{E}\left(\log _{2}(1+\operatorname{SINR})\right) \\
& =\int_{0}^{\infty} \operatorname{Pr}\left\{\log _{2}(1+\operatorname{SINR}>t)\right\} d t \\
& =\int_{0}^{\infty} \frac{\operatorname{Pr}\left\{\operatorname{SINR}>e^{t}-1\right\}}{\log (2)} d t \\
& \stackrel{(a)}{=} \frac{1}{\log (2)} \int_{0}^{\infty} \frac{\operatorname{Pr}\{\operatorname{SINR}>x\}}{(x+1)} d x \\
& =\frac{1}{\log (2)} \int_{0}^{\infty} \frac{p_{c}(x)}{(x+1)} d x
\end{aligned}
$$

Where (a) follows from a variable change $\left(x=e^{t}-1\right)$.

Hence, the throughput is derived by replacing $p_{c}(T)$ (Eq. 4) in equation 22 .

Remark 2: The expectation in eq. 4 is over the p.p. and it is difficult to compute because the probability density function (pdf) of the $r$ - $l$ square p.p. is unknown. Hence, Monte Carlo simulation is used to validate results.

\section{Simulation AND RESUlts}

We consider a 1-tier network composed by LPNs connected by a mesh network. According to [20] which gives guidelines of picocells characteristics, we set $p_{t}=250 \mathrm{~mW}(24 \mathrm{dBm})$, $r=50 \mathrm{~m}$, the sides of squares, and $l=30 \mathrm{~m}$, the sides of sub-squares. By this configuration, the distance between two nodes can not be less than $r-l=20 \mathrm{~m}$. The path loss model considered is given by: $l(r)=r^{-\alpha}$, where $\alpha$ is the path loss 


\begin{tabular}{|c|c|}
\hline Parameters & Values \\
\hline$r$ & $50 \mathrm{~m}$ \\
\hline$l$ & $30 \mathrm{~m}$ \\
\hline Path-loss function & $l(r)=r^{-\alpha}$ \\
\hline$\alpha$ & 3.0 \\
\hline$p_{t}$ & $250 \mathrm{~mW}$ \\
\hline Bandwidth & $10 \mathrm{MHz}$ \\
\hline Noise & Normal $\left(1.0 e^{-11}, 3.76 e^{-11}\right)$ \\
\hline
\end{tabular}

TABLE I: Parameters for the numerical evaluation.

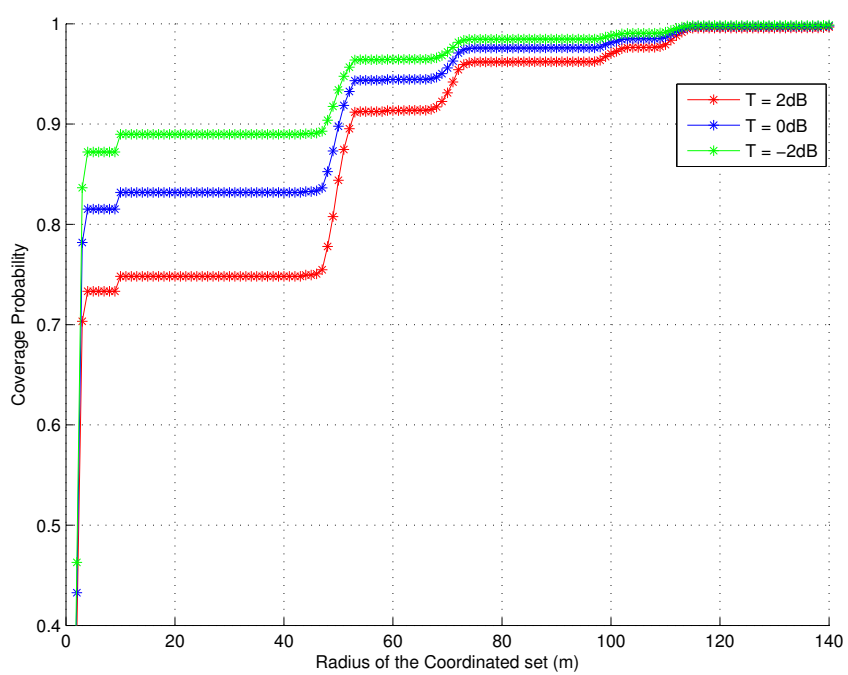

Fig. 4: The coverage probability in function of $d$, the radius of the coordinated set $\mathcal{C}$ for different values of the SINR threshold $T$

exponent. Furthermore, we use a bandwidth of $10 \mathrm{MHz}$. We consider a network composed of 25 squares and a typical user placed in the center of the grid. We take, for analysis, the mean over 1000 realizations of the spatial process. Simulation parameters are summarized in table I.

Figure 4 shows the coverage probability $p_{c}$ of a user placed at the cell edge versus $d$, the radius of the coordinated set. It reflects the impact of the coordinated set size on this performance metric. We plot $p_{c}$ for three values of the SINR threshold, $-2 \mathrm{~dB}, 0 \mathrm{~dB}$ and $2 \mathrm{~dB}$. We observe that the coverage probability increases with the radius of the coordinated set and it is too close to 1 when the radius of the coordinated set exceeds $70 \mathrm{~m}$ (equivalent to 5-6 LPNs in $\mathcal{C}$ ). The value 1 around $120 \mathrm{~m}$ which is equivalent to 7-8 LPNs in the coordination set $\mathcal{C}$. Obviously, the smaller the SINR threshold $\mathrm{T}$ is, the better the coverage is $\left(p_{c}(-2 d B)>p_{c}(0 d B)>p_{c}(2 d B)\right)$.

In figure 5 , we show the coverage probability of the CoMPJT versus the SINR threshold. Simulations are performed for six values of $d$, the ball radius: $30,50,75,100,150$ and $200 \mathrm{~m}$. Figure 5 shows the effect of coordination for these values of $d$ on the coverage. In fact, when $d$ becomes larger, the coordinated set size is increased, the coverage probability is enhanced. This gain can be characterized as follows:

$$
G\left(p_{t}\right)=\frac{\left(P_{c}^{\text {Coordinated set radius }}\right)^{-1}\left(p_{c}\right)}{\left(P_{c}^{\text {Baseline radius }}\right)^{-1}\left(p_{c}\right)}
$$

\begin{tabular}{|c|c|c|}
\hline Baseline radius & Coordinated set radius & Gain \\
\hline \hline $30 \mathrm{~m}$ & $50 \mathrm{~m}$ & $2 \mathrm{~dB}$ \\
\hline $30 \mathrm{~m}$ & $75 \mathrm{~m}$ & $2.5 \mathrm{~dB}$ \\
\hline $30 \mathrm{~m}$ & $100 \mathrm{~m}$ & $2.7 \mathrm{~dB}$ \\
\hline $30 \mathrm{~m}$ & $150 \mathrm{~m}$ & $2.9 \mathrm{~dB}$ \\
\hline $30 \mathrm{~m}$ & $200 \mathrm{~m}$ & $3 \mathrm{~dB}$ \\
\hline
\end{tabular}

TABLE II: The gain of cooperation

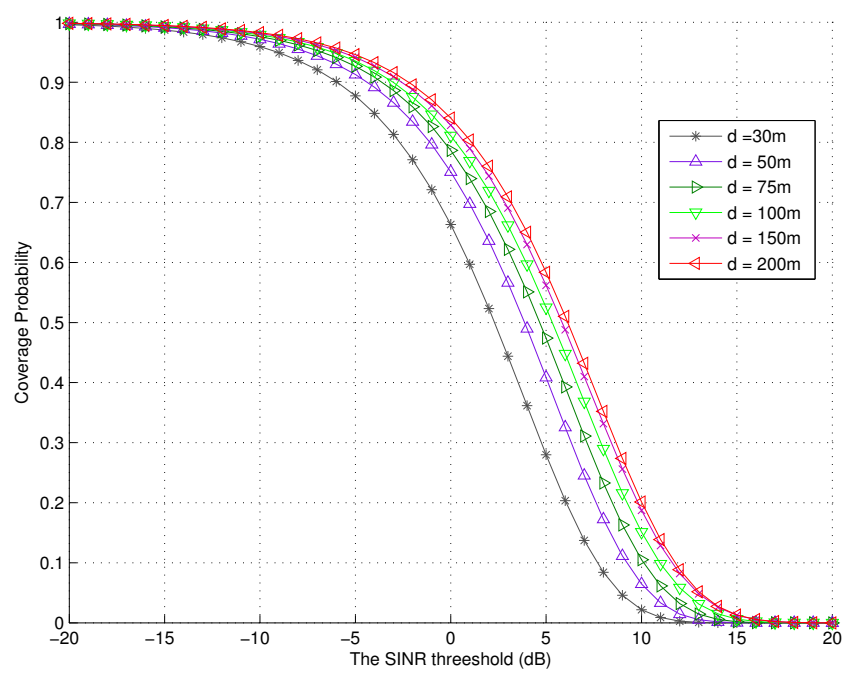

Fig. 5: The coverage probability versus the SINR threshold, for different values of $d$.

where $P_{c}^{\text {Baseline radius }}$ and $P_{c}^{\text {Coordinated set radius }}$ are the coverage probabilities of the $r-l$ square when the user equipment is served by its serving BS and when the radius of $B_{u}$ is $d$, respectively. The case $d=30 \mathrm{~m}$ represents the baseline radius because only the serving BS is inside the coordinated set. $(.)^{-1}\left(p_{c}\right)$ is the SINR threshold value where the coverage probability is set to $p_{c}$. This definition is similar to the coding gain in the coding theory. Table (table II) gives some examples of the gain that we can get from cooperation.

Figure 6 shows the average rate versus the radius of the coordination set for different values of the path loss exponent. The average data rate is an increasing function of number of BSs in $\mathcal{C}$. We can also see that the curves have a step function form. This can be explained by the fact that the rate remains constant until a new BS joint the coordinated set. The transition from one stair to the upper one is done when a new BS joint the coordinated BSs.

For the coverage and the throughput, it is clear from fig. 5 and fig. 6, that after a certain radius of the coordination set, those performance metrics remain constant or slightly improve. This can be explained by the fact that the interference is mainly generated by the neighbor BSs (the 8 squares) surrounding the serving BS.

In a configuration of $5 \times 5$ squares, we have shown for different CoMP sizes that this technique effectively improves the performance in terms of coverage probability and throughput. 


\section{REFERENCES}

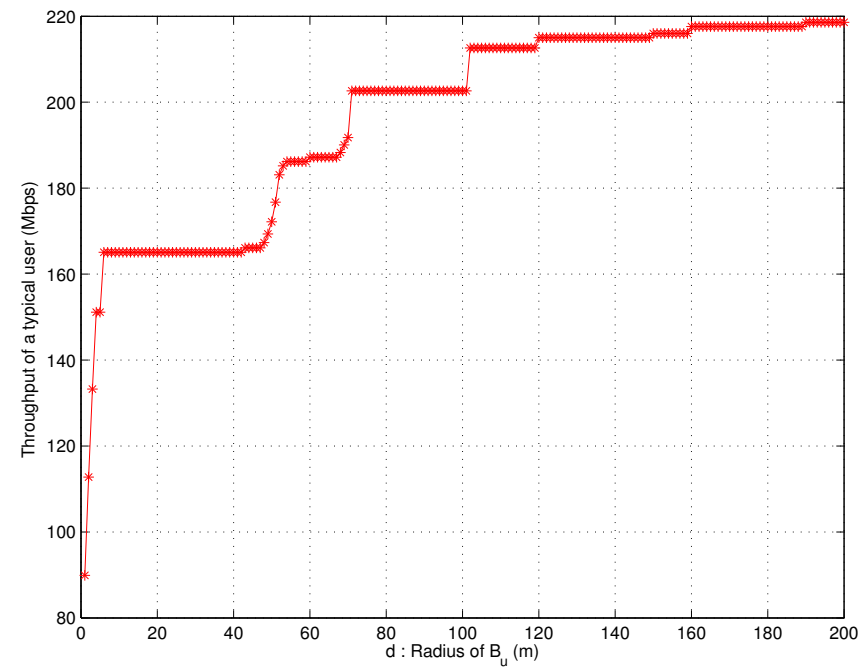

Fig. 6: Throughput of a typical user in function of the coordination set size.

\section{CONCLUSion}

In this paper, we consider a 1-tier network composed of multimode femtocells (LTE/Wi-Fi) linked by a mesh network. It is a cost-efficient solution proposed by cellular operators to offer connectivity service to mobile users in uncovered or poorly covered rural areas. In such deployment, mobile users communications are carried in through the Wi-Fi mesh network to access the macro base station.

We focus our work on the evaluation of the gain that can provide the coordination multipoint joint transmission approach (CoMP-JT) when used to mitigate interference between multimode femtocells. CoMP-JT is a cooperation method where a mobile user receives duplicated signals from a coordinated set of femtocells, in order to limit interference and enhance network performance mainly the coverage probability and the throughput. We use the $r-l$ square point process proposed earlier to model the multimode femtocells position. We derive the expressions of the coverage probability $p_{c}$ and the rate $R$ of a typical user on the downlink. By simulation, we showed that increasing the coordination set size improves the performance of our system. Furthermore, we conclude that after a certain coordination set radius (d) threshold it is inefficient to add more BSs to the coordination set $\mathcal{C}$.

Our future work is investigating the probability density function (pdf) of $r$ - $l$ square p.p. in order to develop a closedform for the coverage probability and the throughput.

\section{ACKNOWLEDGEMENT}

This work is supported by the French project LCI4D of the Systematic Cluster, Paris Region Systems \& ICT Cluster (http://www.systematic-paris-region.org/).
[1] International Telecommunication Union, "Measuring the information society 2013," 2013.

[2] M. Bennis, M. Simsek, A. Czylwik, W. Saad, S. Valentin, and M. Debbah, "When cellular meets WiFi in wireless small cell networks," Communications Magazine, IEEE, vol. 51, no. 6, 2013.

[3] H. Dhillon, R. Ganti, F. Baccelli, and J. Andrews, "Modeling and analysis of k-tier downlink heterogeneous cellular networks," Selected Areas in Communications, IEEE Journal on, vol. 30, no. 3, pp. 550-560, April 2012.

[4] R. Heath and M. Kountouris, "Modeling heterogeneous network interference," in Information Theory and Applications Workshop (ITA), 2012, Feb 2012, pp. 17-22.

[5] M. Di Renzo, A. Guidotti, and G. Corazza, "Average rate of downlink heterogeneous cellular networks over generalized fading channels: A stochastic geometry approach," Communications, IEEE Transactions on, vol. 61, no. 7, pp. 3050-3071, July 2013.

[6] B. Blaszczyszyn and H. P. Keeler, "Studying the SINR process of the typical user in poisson networks by using its factorial moment measures," CoRR, vol. abs/1401.4005, 2014.

[7] S. Akoum and R. W. H. Jr., "Interference coordination: Random clustering and adaptive limited feedback," CoRR, vol. abs/1210.6095, 2012.

[8] F. Baccelli, B. Blaszczyszyn, and P. Muhlethaler, "Stochastic analysis of spatial and opportunistic aloha," Selected Areas in Communications, IEEE Journal on, vol. 27, no. 7, pp. 1105-1119, September 2009.

[9] T. Shirai and N. Miyoshi, "A cellular network model with ginibre configurated base stations," Advances in Applied Probability, 2013.

[10] I. Nakata and N. Miyoshi, "Spatial stochastic models for analysis of heterogeneous cellular networks with repulsively deployed base stations," Performance Evaluation, vol. 78, no. 0, pp. 7 - 17, 2014. [Online]. Available: http://www.sciencedirect.com/science/article/ pii/S0166531614000546

[11] N. Deng, W. Zhou, and M. Haenggi, "The Ginibre Point Process as a Model for Wireless Networks with Repulsion," ArXiv e-prints, Jan. 2014.

[12] A. Busson, L. Zitoune, V. Vèque, and B. Jabbari, "Outage Analysis of Integrated Mesh LTE Femtocell Networks," Apr. 2014, submitted to Globecom'14 LCI4d FUI Project. [Online]. Available: http://hal-supelec.archives-ouvertes.fr/hal-00979627

[13] R. Tanbourgi, S. Singh, J. G. Andrews, and F. Jondral, "A tractable model for non-coherent joint-transmission base station cooperation," CoRR, vol. abs/1308.0041, 2013.

[14] G. Nigam, P. Minero, and M. Haenggi, "Coordinated multipoint in heterogeneous networks: A stochastic geometry approach," in IEEE GLOBECOM Workshop on Emerging Technologies for LTE-Advanced and Beyond 4G (GLOBECOM-B4G'13), (Atlanta, GA), Dec. 2013.

[15] P. Xia, C.-H. Liu, and J. G. Andrews, "Downlink coordinated multi-point with overhead modeling in heterogeneous cellular networks," CoRR, vol. abs/1210.5503, 2012.

[16] B. Matern, "Spatial variation," Meddelanden fran Statens Skogsforskningsinstitut, vol. 49, No. 5, 1960.

[17] A. M. Ibrahim, T. ElBatt, and A. El-Keyi, "Coverage probability analysis for wireless networks using repulsive point processes," in Personal Indoor and Mobile Radio Communications (PIMRC), 2013 IEEE 24th International Symposium on, Sept 2013, pp. 1002-1007.

[18] Y. Yifan, R. Yun, L. MingQi, S. Bin, and S. RongFang, "Achievable rates of coordinated multi-point transmission schemes under imperfect csi," In Proc. IEEE ICC, pp. 1-6, 2011.

[19] S. Berger, Z. Lu, R. Irmer, and G. Fettweis, "Modelling the impact of downlink comp in a realistic scenario," in Wireless Communications and Networking Conference (WCNC), 2013 IEEE, April 2013, pp. 39323936.

[20] 3GPP TR 136931 V9.0.0 (2011-05) (2011-05), "LTE; Evolved Universal Terrestrial Radio Access (E-utra); radio frequency (RF) requirements for LTE pico node B.” 